\title{
Sonoporation: From the lab to human clinical trials
}

\author{
Spiros Kotopoulis* ${ }^{*}$, Georg Dimcevski*, Bjørn Tore Gjertsen ${ }^{\ddagger}$, Odd Helge Gilja*§, \\ Emmet Mc Cormack ${ }^{\ddagger}$ and Michiel Postema ${ }^{\dagger}$ \\ *National Centre for Ultrasound in Gastroenterology, Haukeland University Hospital, \\ Jonas Lies vei 65, 5021 Bergen, Norway \\ ${ }^{\dagger}$ Department of Physics and Technology, University of Bergen, Allégaten 55, 5007 Bergen, Norway \\ ${ }^{\ddagger}$ Department of Clinical Science, University of Bergen, Jonas Lies vei 65, 5021 Bergen, Norway

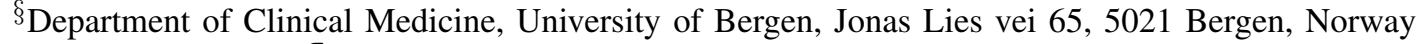 \\ \Corresponding author. Email: Spiros.Kotopoulis@uib.no
}

\begin{abstract}
Therapeutic ultrasound has been in use for over 70 years but has primarily been a thermal modality. Sonoporation, the use of ultrasound and stable gas microbubbles in the size range of $2-10 \mu \mathrm{m}$ to form transient pores in cell membranes, has been of great interest in the past 15 years. This technique could be used to improve the delivery of current drugs in very localised regions. There are several phenomena behind sonoporation that all occur non-exclusively: push, pull, jetting, inertial cavitation, shear and, translation. Pre-clinical work has shown that sonoporation can be used to reduce primary tumour burden and inhibit metastatic development. Our clinical trial showed that ultrasound in combination with microbubbles and chemotherapy can effectively double the number of chemotherapy cycles patients can undergo, meaning that the patients were healthier for a longer period of time. Nevertheless, sonoporation is still in its infancy and there is vast room for improvement in both the areas of microbubbles and ultrasound.
\end{abstract}

\section{INTRODUCTION}

Therapeutic ultrasound has been in use since the early 1940 s [1], primarily as method to heat deep tissue to promote healing. Nevertheless, in the past 15 years, due to advancements in technology, new ultrasound-based therapeutic modalities have surfaced; specifically High Intensity Focused Ultrasound (HIFU) and sonoporation. HIFU is used to heat targeted tissue in very specific regions to such a high temperature that thermal necrosis occurs. This is often done with the aid of Magnetic Resonance Imaging to visualise the target tissue and measure the thermal deposition [2]. Sonoporation on the other hand aims to solve the problem of time and space controlled drug delivery [3]. One of the major barriers in effective drug delivery is the cell membrane; it acts as wall selectively choosing what to allow in and out of the cell [4]. Sonoporation can be defined as the the transient formation of pores in a cell membrane due to ultrasound or a combination of ultrasound and microbubbles. By forming pores in the cell membranes we can effectively bypass this barrier that is the cell membrane, resulting in highly efficient targeted drug delivery. In our work we explore the phenomenon of sonoporation, from the lab bench all the way to the World's first clinical trials.

\section{MicRobubBles IN A SAFE CLINICAL SETTING}

The first clinical use of microbubbles was reported in 1969 [5]. Microbubbles in the blood pool are used to improve the signal-to-noise ratio or contrast of blood when imaged
TABLE I

CLINICALLY APPROVED ULTRASOUND CONTRAST AGENTS.

\begin{tabular}{|c|c|c|c|c|}
\hline $\begin{array}{l}\text { Contrast } \\
\text { agent }\end{array}$ & Shell & Gas & $\begin{array}{l}\text { Size range } \\
\varnothing(\mu \mathrm{m})\end{array}$ & Manufacturer \\
\hline Optison $^{\mathrm{TM}}$ & Albumin & $\mathrm{C}_{3} \mathrm{~F}_{8}$ & $2.2-4.5$ & GE Healthcare \\
\hline SonoVue ${ }^{\circledR}$ & Lipid & $\mathrm{SF}_{6}$ & 2.5 & Bracco Int. \\
\hline Definity ${ }^{\circledR}$ & Lipid & $\mathrm{C}_{3} \mathrm{~F}_{8}$ & $1.1-3.3$ & $\begin{array}{l}\text { Lantheus Medical } \\
\text { Imaging }\end{array}$ \\
\hline Sonazoid ${ }^{\circledR}$ & Lipid & $\mathrm{C}_{4} \mathrm{~F}_{10}$ & $1.9-2.4$ & $\begin{array}{l}\text { Daiichi } \\
\text { Pharmaceutical Co. }\end{array}$ \\
\hline
\end{tabular}

using B-mode sonography. Hence, these microbubbles are also known as ultrasound contrast agents. Their sizes range between $2-5 \mu \mathrm{m}$, typically with over $95 \%$ below $10 \mu \mathrm{m}$ so that the microbubbles can pass through the lung capillaries. Microbubbles, like most physical objects, have a resonant frequency; a frequency where its oscillation amplitude is maximal. Similar to a bell, when a force strikes it, the microbubble will volumetrically oscillate and generate sound at its resonance frequency. For a free gas bubble this frequency can be approximated by [6]:

$$
f_{r} \approx \frac{6.5}{D}
$$

where $f_{r}$ is the resonant frequency and $D$ is the diameter of the bubble. From this we can see that a $2.5 \mu \mathrm{m}$ gas bubble will resonate at approximately $2.6 \mathrm{MHz}$, a typical diagnostic ultrasound frequency. Free gas microbubbles are inherently unstable, and dissolve almost instantly. For this reason clinical diagnostic ultrasound contrast agents typically have a lipid or albumin shell with a slowly diffusing gas core, increasing stability, allowing for efficient clinical imaging. Table I summarises clinically approved ultrasound contrast agents and their contents.

In clinical diagnostic ultrasound imaging, the Mechanical Index (MI) gives an indication of the mechanical damage possible due to inertial cavitation when an ultrasound contrast agent is present. It is defined by: 


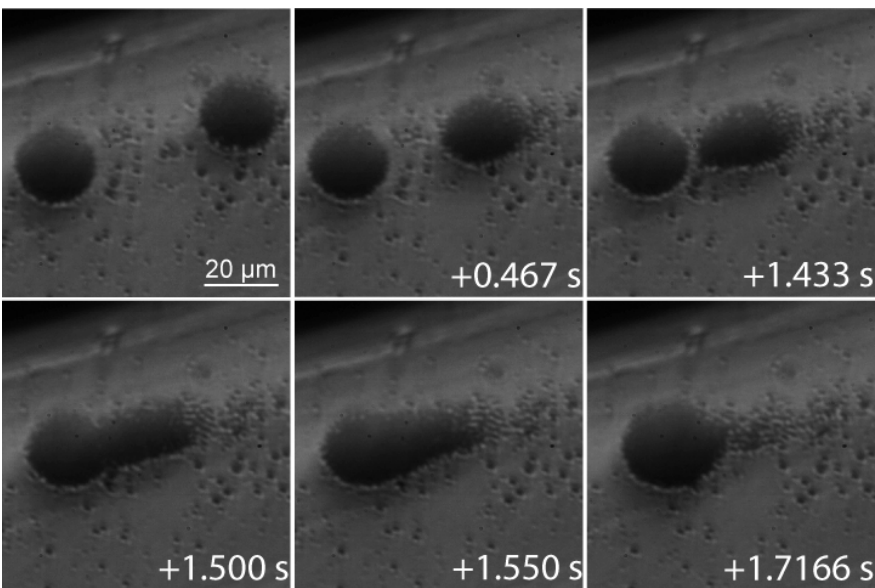

Fig. 1. Photo micrographs depicting clusters of Definity ${ }^{\circledR}$ microbubbles attracting and merging in under 2 seconds.

$$
\mathrm{MI}=\frac{p^{-}}{\sqrt{f_{\mathrm{c}}}},
$$

where $p^{-}$is the peak-negative acoustic pressure in-situ measured in $\mathrm{MPa}$, and $f_{\mathrm{c}}$ is the centre frequency in $\mathrm{MHz}$. An $\mathrm{MI}<0.3$ is considered safe, an MI between 0.3 and 0.7 is considered moderately safe as there is a risk of damage to neonatal lung tissue or intestine, and an MI $>0.7$ there is a high risk of inertial cavitation of the ultrasound contrast agents, and a theoretical risk of cavitation formation without ultrasound contrast agents present [7]. For this reason, in all our work, we attempt to work at $\mathrm{MI} \leq 0.2$, ensuring no destructive and currently uncontrollable inertial cavitation is present and allowing for faster transition from lab to clinic.

The first step in being able to induce localised sonoporation was to attempt to control the location of the microbubbles [8]. A custom made experimental setup incorporating a $2.2 \mathrm{MHz}$ ultrasound transducer, a synthetic $\varnothing 200-\mu \mathrm{m}$ capillary and a high-speed camera was built on top of an inverted microscope. Continuous-wave ultrasound with centre frequencies of $2-\mathrm{MHz}$ and 7-MHz with peak-to-peak acoustic pressures of $20 \mathrm{kPa}$ were used in conjunction with Definity ${ }^{\circledR}$ ultrasound contrast agent. Upon insonation, the microbubbles were seen to attract each other forming small spherical clusters of microbubbles. These clusters of 1-2 thousand microbubbles would form within several seconds, and were spaced $1 / 4 \lambda$ apart. As the attracted microbubbles were oscillating in phase, when continuing sonication, the clusters were attracted to each other, forming even larger clusters (c.f. Fig. 1). When increasing the acoustic pressure the clusters could be radiated towards the membrane wall. These results indicated that if necessary, microbubbles could be accumulated to specific regions and radiated toward a vascular wall.

\section{In-vitro WORK}

In-vitro work has been the hallmark for evaluating cellular effects for years. In our work, we attempted to understand why specific acoustic conditions were optimal for increased drug delivery.

As can be expected, injecting a gas bubble into the blood stream, that generates a detectable acoustic force, may have some inherent side-effects. In 1997, upon studying such side-effects [9] and [10] showed that ultrasound in combination with microbubbles could increase the uptake of fluorescent genes.

Following this exciting discovery, several research groups, both experimental and theoretical rushed to understand the physical behaviours behind this increased uptake. It accepted that the oscillatory behaviour of the microbubbles under sonication forms small transient pores in the cell membrane, yet the question of how still remained.

Hence, the next step was to attempt to understand the physical mechanisms of microbubble-cell interaction using optimised acoustic conditions. Whilst a vast majority of high-speed imaging is performed at several million frames per second, we attempted to record at slower frame rates giving us longer imaging duration but with sufficient temporal resolution to track microbubble motion. For this work we used immortalised cervical cancer cells (HeLa cells). $1.6 \times$ $10^{6}$ cells were seeded into an OptiCell ${ }^{\mathrm{TM}}$ and incubated for 24 hours. Following incubation, low concentrations of $\mathrm{DiD}$ lipophilic fluorescent probe (Vybrant ${ }^{\mathrm{TM}}$ Molecular Probes) tagged lipid shelled microbubbles, Definity ${ }^{\circledR}$, SonoVue ${ }^{\circledR}$ or MicroMarker $^{\mathrm{TM}}$ (Bracco) were injected into the OptiCell ${ }^{\mathrm{TM}}$. Concentrations of 1 bubble to 1 cell were aimed for. The OptiCell $^{\mathrm{TM}}$ was then placed in a water bath cell-side up, ensuring the floating bubbles were in contact with the cells. Two ultrasound frequencies were evaluated: $1.0 \mathrm{MHz}$ and $6.6 \mathrm{MHz}$ with duty cycles of $40 \%$ and $50 \%$, respectively. The MI for both frequencies was $\leq 0.2$ and acoustic intensity ( $\mathrm{I}_{\mathrm{SPTA}}$ ) was $\ll 1 \mathrm{~W} / \mathrm{cm}^{2}$. Imaging was performed using a high-magnification, high-numerical aperture (NA) setup using a $60 \times / 0.90-\mathrm{NA}$ objective or using a confocal fluorescence microscope with a $40 \times / 1.30-\mathrm{NA}$ objective. Following sonication, a range of phenomena was observed, including bubbles hammering back and forth into cells [11], to complete bubble translation into a cell. To validate if the microbubble had truly translated into the cell, 3D confocal fluorescence imaging was performed. Results showed that the lipid bonded fluorescent probe was then within the cell. To exclude the chance of endocytosis, experiments were re-done with endocytosis inhibitors where no change in localised microbubble translation was seen.

In conclusion, to date there are several non-exclusive phenomena that can be directly related to this increased up take [12]: Push and pull, where the microbubble near, or in contact with the cell, stretches the bilayer membrane opening pores. Jetting, when a bubble collapses asymmetrically, making a needle-like point towards the cell. Streaming, where the fluid flow around the bubble near a cell is strong enough to shear the membrane apart. Translation, where a microbubble with a therapeutic load can be forced inside a cell. Inertial cavitation, where the violent formation 


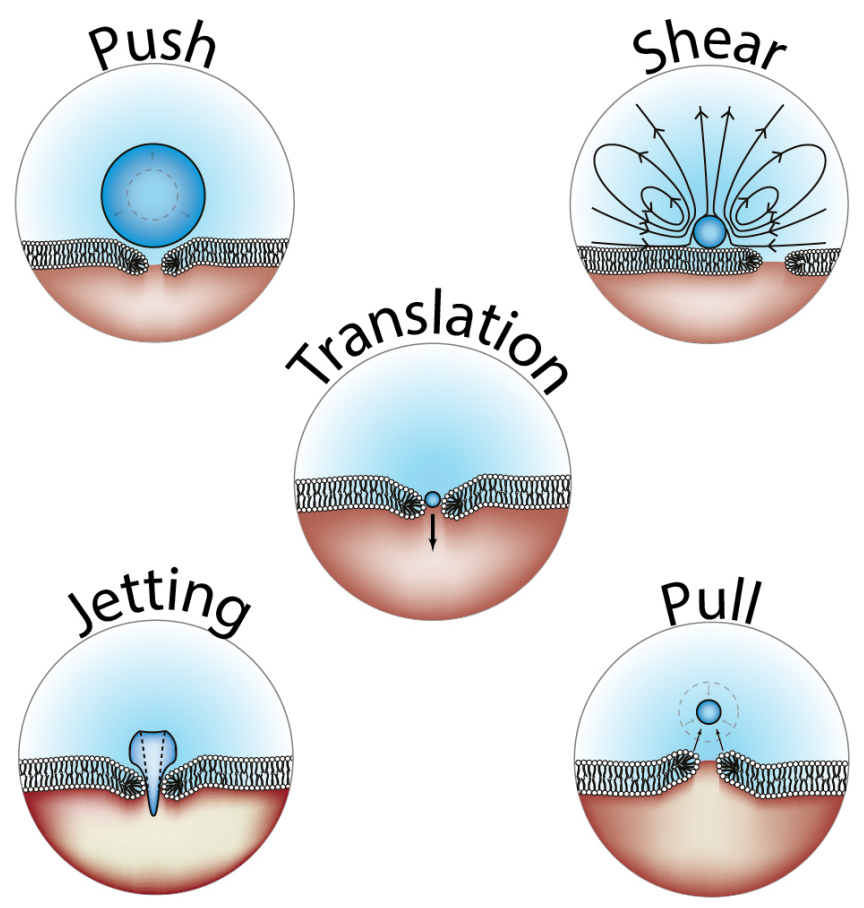

Fig. 2. Illustration depicting five of the sonopration mechanisms.

and destruction of bubbles generates shock-waves damaging everything in the local area. It is important to understand that all these phenomena are non-exclusive, and each and all have advantages and disadvantages. In addition, when sonoporation will occur most effectively when exciting the microbubbles at their resonance frequency. Figure 2 illustrates five of the sonoporation mechanisms that induce transient cell membrane porosity. In addition to these phenomena there are several controversial yet thought-provoking theories regarding the acoustic activity of the cell membranes themselves [13].

\section{PRE-CLINICAL WORK}

Pre-clinical mouse work was performed to evaluate the effect of sonoporation in combination with a chemotherapeutic on an orthotopic pancreatic ductal carcinoma (PDAC) model [14].

The orthotopic xenograft model was developed by injecting $1 \times 10^{6}$ bioluminescent human PDAC cells (MIA PaCa-2 ${ }^{\text {luc }}$ ) into the head of the pancreas of immunodeficient (NOD-scid IL2r $\gamma^{\text {null }}$ ) mice. The bioluminescent cells allowed for 2D full body imaging helping visualise the spread of the tumour cells. The 3D development of the primary tumour was measured using high-resolution 3D ultrasound (MS250 probe and Vevo 2100, VisualSonics Inc, Ontario, Canda).

Mice were imaged and treated once weekly using Gemzar (Eli Lilly and Company, Indianapolis, IN) the current best chemotherapeutic for treating PDAC, or Gemzar in combination with ultrasound and SonoVue ${ }^{\circledR}$ microbubbles. Acoustic conditions remained the same as in the in-vitro work, where bubbles were forced into the cells, i.e., $1-\mathrm{MHz}, 40$

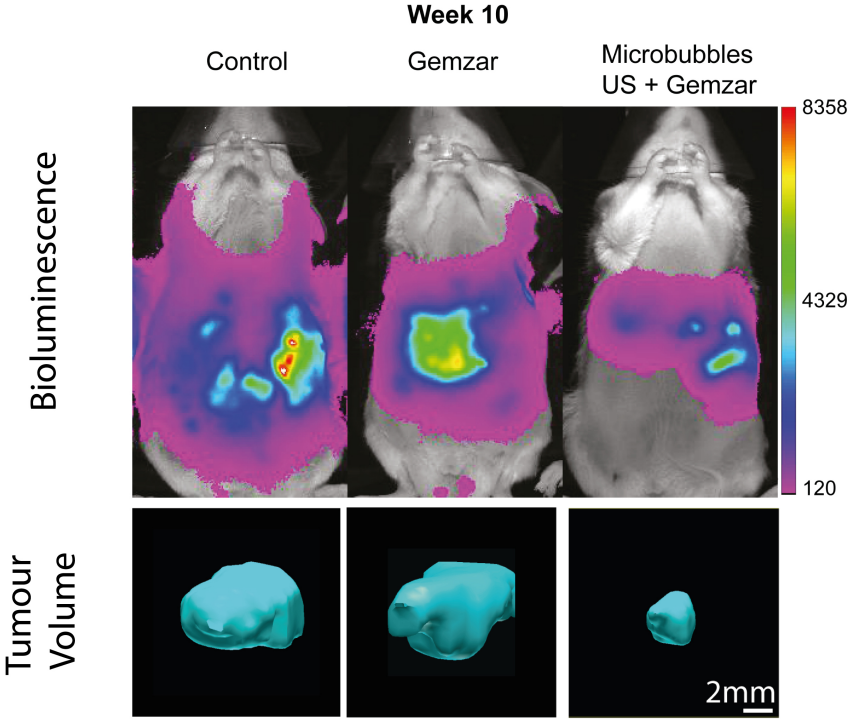

Fig. 3. Bioluminesence imaging and tumour volume of mice with orthotopic pancreatic cancer treated with Gemzar alone or Gemzar with microbubbles and ultrasound.

cycles, MI $=0.2$, with a $40 \%$ duty cycle. Treatment was started 3 weeks after xenografting.

After only two treatments, i.e., 5 weeks after xenografting, a statistical significant difference in tumour volume was seen between the sonoporated group and the chemotherapy alone group. Figure 3 shows the bioluminescent images and tumour volumes 10 weeks after xenografting (after six treatments). By week 10, the primary tumour in the sonoporation group was nearly 4 times smaller than the control and chemotherapy alone tumour. In addition the metastatic spread was limited to the liver in the sonoporation group whereas the Gemzar and control group showed whole body metastatic spread. In conclusion, translating the identical acoustic conditions seen to force microbubbles into cells in vitro resulted in primary tumour inhibition and delayed metastatic spread when combining a chemotherapeutic.

\section{Clinical trials}

In many cases pre-clinical work does not translate well into the clinic. In our work here we perform, to our knowledge, the world first clinical trial on sonoporation [15].

A total of five patients with PDAC were treated using Gemzar, SonoVue ${ }^{\circledR}$ and ultrasound. The chemotherapeutic was administered in accordance to the manufacturer's guidelines; infusion over 30 minutes. At the completion of the infusion, the concentration of the chemotherapeutic was maximal in the blood. At this point sonoporation was initiated. Ultrasound was generated using a clinical diagnostic scanner (GE LOGIQ $9+4$ C probe, GE Healthcare, Waukesha, WI), with optimised acoustic output conditions. The clinical diagnostic machine allowed for simultaneous imaging and treatment of the primary tumour. The output frequency was $1.8 \mathrm{MHz}$ with a duty cycle of $1 \%$. To compensate for the 
reduced duty cycle the ultrasound treatment lasted $31.5 \mathrm{~min}$. SonoVue ${ }^{\circledR}$ were injected intravenously every 3.5 minutes resulting in 9 doses of $0.5 \mathrm{~mL}$ each over 31.5 minutes.

To evaluate the effect of the treatment, we compared the number of chemotherapy cycles our patients could undergo versus 80 historical patients treated at the same hospital. The healthier the patients are, the more treatment cycles they can undergo. The historical group was able to receive $9 \pm 6$ treatment cycles. The patients treated with sonoporation were able to undergo almost double the amount of treatment with $16 \pm 7$ cycles of chemotherapy. It is important to note that several patients were removed from the study due to the efficacy of the treatment. These patients would be able to go substantially more cycles, hence the treated cycles number is artificially low.

Maximum tumour diameters were measured using CT imaging from independent radiologists. The primary tumour growth was inhibited and in some cases, even reduced.

In conclusion, the clinical trials showed that there are no immediate adverse side effects to sonoporation, and that sonoporation can help increase the efficacy of the treatment.

\section{FUTURE WORK}

Whilst sonoporation has shown success in the lab and in the clinic, there is still a lot of room for improvement. To date, sonoporation has primarily been performed by combining existing materials and technologies aimed for other tasks, for example the ultrasound contrast agents and clinical diagnostic machines are designed for imaging not treatment. Both these aspects need to be drastically improved.

\section{A. Microbubbles}

In the area of microbubbles, new agents need to be developed that can carry a therapeutic load to the target region. These bubbles need to be highly acoustically sensitive, and be able to release their load on demand. Current concepts allow loading inside the shell, or on the surface of the shell, hence only small loads can be carried. New methods and concepts are needed that allow for larger volumes of therapeutic loads and specific release mechanisms. Such a method would only treat a localised region, and would have minimal affect on metastasis, for this reason, it may be ideal to combine sonoporation with traditional chemotherapy, ensuring maximum affect on both primary tumour and metastasis.

\section{B. Ultrasound}

Clinical diagnostic machines are highly specialised and have inverse acoustic properties than those needed for sonoporation. Sonoporation requires a large area to be treated, so a large footprint probe, that can treat a whole tumour is necessary. In addition, longer duty cycles are required to continuously excite the microbubbles in the target region increasing the cellular permeability.

\section{CONCLUSION}

Although sonoporation was discovered only in 1997 and clinical trials were first done from 2013, we have shown that there is great potential in sonoporation. In-vitro results have shown that microbubbles can be forced into cells, whereas pre-clinical mice studies under identical conditions have shown to reduce primary tumour burden and metastatic development. Our clinical trial showed that combining sonoporation and chemotherapy can help the patients to undergo more chemotherapy cycles, presumably prolonging survival.

\section{ACKNOWLEDGEMENTS}

This study has been supported by MedViz (http://medviz.uib.no/), an interdisciplinary research cluster from Haukeland University Hospital, University of Bergen, and Christian Michelsen Research AS. The authors would like to thank The Michelsen Centre for Industrial Measurement Science and Technology for their support throughout this project.

\section{REFERENCES}

[1] V. J. Robertson and K. G. Baker, "A review of therapeutic ultrasound: effectiveness studies," Phys Ther, vol. 81, no. 7, pp. 1339-1350, 2001.

[2] K. Hynynen, "MRI-guided focused ultrasound treatements," Ultrasonics, vol. 50, no. 2, pp. 221-229, 2010.

[3] B. Geers, H. Dewitte, S. C. De Smedt, and I. Lentacker, "Crucial factors and emerging concepts in ultrasound-triggered drug delivery," J Control Release, vol. 164, no. 3, pp. 248-255, 2012.

[4] C. Peetla, S. Vijayaraghavalu, and V. Labhasetwar, "Biophysics of cell membrane lipids in cancer drug resistance: Implications for drug transport and drug delivery with nanoparticles." Adv. Drug Deliv. Rev., vol. 65, no. 13-14, pp. 1686-98, Nov. 2013.

[5] R. Gramiak and P. M. Shah, "Echocardiography of the aortic root," Invest. Radiol., vol. 3, no. 5, pp. 356-366, 1968.

[6] M. Postema, A. Bouakaz, M. Versluis, and N. de Jong, "Ultrasound-induced gas release from contrast agent microbubbles," IEEE Trans. Ultrason. Ferroelectr. Freq. Control, vol. 52, no. 6, pp. 1035-1041, 2005.

[7] G. ter Haar, "Safety and bio-effects of ultrasound contrast agents," Med. Biol. Eng. Comput., vol. 47, no. 8, pp. 893-900, 2009.

[8] S. Kotopoulis and M. Postema, "Microfoam formation in a capillary," Ultrasonics, vol. 50, no. 2, pp. 260-268, 2010.

[9] S. Bao, B. D. Thrall, and D. L. Miller, "Transfection of a reporte plasmid into cultured cells by sonoporation in vitro," Ultrasound Med. Biol., vol. 23, no. 6, pp. 953-959, 1997.

[10] Unger and E, "SonoPoration and Gene Delivery with Acoustically Active Carriers," p. S2, 1997.

[11] P. Marmottant and S. Hilgenfeldt, "Controlled vesicle deformation and lysis by single oscillating bubbles," Nature, vol. 423, pp. 153-156, 2003.

[12] M. Postema, S. Kotopoulis, A. Delalande, and O. H. Gilja, "Sonoporation: Why microbubbles create pores," Ultraschall Med., vol. 33, no. 1, pp. 97-98, 2012.

[13] B. Krasovitski, V. Frenkel, S. Shoham, and E. Kimmel, "Subcellular sonophores: ultrasound induced intramembrane cavitation," Proc. Nat. Acad. Sci., vol. 108, no. 8, pp. 3258-3263, 2011.

[14] S. Kotopoulis, A. Delalande, M. Popa, V. Mamaeva, G. Dimcevski, O. H. Gilja, M. Postema, B. T. Gjertsen, and E. Mc Cormack, "Sonoporation-enhanced chemotherapy significantly reduces primary tumour burden in an orthotopic pancreatic cancer xenograft," Mol. Imaging Biol., vol. 16, no. 1, pp. 53-62, 2014.

[15] S. Kotopoulis, G. Dimcevski, O. H. Gilja, D. Hoem, and M. Postema, "Treatment of human pancreatic cancer using combined ultrasound, microbubbles, and gemcitabine: A clinical case study," Med. Phys., vol. 40, no. 7, pp. 72 902-72909, 2013. 